$\xi_{1}$

\title{
The Technology of the Learning Process Management Expert System in Higher Educational Institutions
}

\author{
Batyrkhan Kuzenbaev ${ }^{1}$, Rosamgul Niyazova ${ }^{2}$, Ayzhan Kuzenbaeva ${ }^{3}$ \\ ${ }^{1}$ Corresponding author: PhD at the Informatics and information security department, L.N.Gumilyov Eurasian National University \\ (Astana city, Satpaeva str. 2,010000,Kazakhstan); kbatyrkhan@yahoo.com \\ ${ }^{2}$ Candidate of technical sciences, Associate Professor at Informatics and information security department, L.N.Gumilyov Eurasian \\ National University (Astana city, Satpaeva str. 2, 010000, Kazakhstan). \\ ${ }^{3}$ Senior Lecturer, Information Systems department at Kostanay State University A. Baytursynov (Kostanay city, Baitursynov str. 47, \\ 110000, Kazakhstan); Ayzhan.81@ mail.ru
}

\begin{abstract}
The present paper considers the development of learning process management expert system in higher educational institutions, based on the ontological approach. The purpose of research is an improve the effectiveness of decision making during the management of learning processes by using intelligent management methods and modern approaches to knowledge modelling. The methodology of solving the set task is based on models and methods of knowledge representation usage, the artificial intelligence theory, expert methods of decisionmaking and general theoretical principles of the control theory, and the theory of decision-making. Authors conducted a qualitative analysis of the domain knowledge, which allowed distinguishing and formalizing main concepts and relations between them. Authors suggested a methodological approach to the construction of the learning process management system, which allows implementing knowledge-based approaches to the development of information systems in the domain knowledge of learning process management. Authors also developed the structure and formal description of the ontology of the learning process in higher educational institutions, which allows reusing the suggested solutions. Research results were implemented in the expert information system; they can be used in practice in learning process management in higher educational institutions.
\end{abstract}

Keywords: expert system; learning process, ontology; higher educational institution; management; knowledge base.

\section{Introduction}

In modern conditions, managerial activity is a crucial factor for the functioning and development of an organization. Efficient management is a valuable resource of organization. Consequently, improving the efficiency of managerial activity improves the activity of an organization overall.

The current quick rate of technological changes requires the adjustment of approaches to the training of future specialists. The expediency of adequate computer support of decision making during education in modern conditions causes growth of information flow for further processing and constant variation of the academic load.

The achievement of an appropriately high level of education requires intensification and interactivity, which is possible with the application of information technologies. The introduction of information technologies into the learning process significantly simplifies the search for and the transfer of large volumes of information, as well as improves the speed of information flows of knowledge, based on the accumulated and modern technological experience. The use of modern information technologies improves the quality of the learning process, since they enhance the educational effect of applying innovative pedagogical programs and methods.
The necessity of using information technologies during the organization of the learning process is determined by the fact that these technologies provide teachers with new possibilities of efficiently organizing education, with a view to achieving the maximum level of academic performance, allow planning scientific and research work, which is necessary for the analysis of complex problems, etc.

The use of knowledge management technologies allows for a flexible adjustment of data storage schemes, adaptation of the information system behavior and the means of information display to the changing requirements of system users. The learning process management in higher educational institutions (LPM HEI) is conducted with a view to preventing inconsistencies and ensuring the planned graduation of bachelors who meet state standards.

The topicality of this study is determined by the need for improving the quality of higher educational institutions' learning process management system by developing methods, which allow adequately evaluating and purposefully projecting the improvement of characteristics onto the learning process management with incomplete or semi-structured background information.

The use of information technologies and artificial intelligence for the automation of educational system management is expedient, which is proved by scientific works. In particular, research papers by 
Webster [12], Liebowitz \& Frank [13], Moore [14], Jones \& Sallis [15] address the issues of implementing intelligent components into education management systems. Modern achievements in the automation of learning process, the development of educational systems, based on the artificial intelligence theory, are based on the scientific achievements of Rennie \& Morrison [16], Al-Qahtani \& Higgins [17], Bogino, Levchuk, \& Petrov [2] and others.

According to Izvozchikov [4], information technologies in learning are the methodology and technology of the learning and educational process with the use of cutting-edge electronic learning means, first of all, computers. Maystrenko \& Maystrenko [6] describe information technologies (IT) as a process that uses a set of means and methods of gathering, processing, and transferring data (background information), in order to obtain new information on the state of the object, process, or phenomenon (information product). The purpose of using IT is the generation of information for its analysis by a human and the making of decisions, based on such information.

IT, used in educational institutions, can be divided into the ones used for the management of the educational institution and the ones used for the organization of learning process [20].

The purpose of this study is to improve the efficiency of decisionmaking in learning process management by developing an expert system and implementing knowledge-based models and methods into higher educational institutions' managerial practice.

The research object is the learning process in higher educational institutions.

The research subject is the information technology of creating a learning process management expert system in higher educational institutions.

The theoretical and methodological bases of development of a decision-making support system in the learning process management system in higher educational institutions consist of systems analysis methods and decision-making theory methods. The following methods are used to complete the set objectives: ontology construction methods and mathematical statistics methods for the construction of learning object models; decision-making theory methods for constructing evaluation models; information theory methods for the evaluation of the functional effectiveness of the system; unified language of ontological knowledge representation.

\section{Methods}

The main objective of the learning process management system in higher educational institutions is the improvement of the quality of education by means of efficient and coordinated organization of work with the possibility of control, analysis, and adjustment of managerial decisions, based on objective results of continuous monitoring of the quantitative and qualitative level of knowledge of teachers and workers. Since, at present, systems of automated test checking are an efficient instrument of knowledge evaluation, the scientific problem of intellectualization of methodological and technological testing instruments by developing and creating expert support systems of decision-making in learning process management in higher educational institutions, based on them, is relevant.

An expert system is a computer program that shows a level of knowledge, equal to that of a human expert in a certain field. In general, such a field is strictly limited. However, the number of applications is vast. This includes speech understanding, image analysis, weather forecast, crop yield estimation, medical diagnostics, development of integrated circuits, funding, air traffic control, battle management, etc.

In order to conduct an examination, the computer program should be capable of solving problems by means of inference and obtaining sufficiently valid results. The program should have an access to a knowledge base. It should also draw conclusions, based on the information, contained in the knowledge base, during consultations. Some expert systems can also use new information, added during the consultation. Thus, the expert system consists of three parts:

1. Knowledge base (KB).

2. Output mechanism (OM).

3. User interface system (UIS).

The practical value of any knowledge-based system primarily depends on the used knowledge base of the domain knowledge. Consequently, one of the important objectives during the development of such systems is the formation of knowledge bases the obtainment of knowledge from an expert in a given field, its formalization and representation in a computer-readable form. At present, specialized knowledge base editors are used for this purpose. Such an editor allows an expert to input knowledge of terms in his domain knowledge.

"LPM HEI" knowledge base was developed using ontological approach.

The use of an ontology as an instrument of organization and visualization of knowledge allows ensuring a system approach to the modelling of the domain knowledge and working with it. This achieves:

- continuity - ontology is a holistic view of domain knowledge;

uniformity - the material, presented in a unified form, is significantly more comprehensible and reproducible;

scientific character - construction of an ontology allows restoring the lacking logical connections in their entirety.

It is expedient to use a set of concepts in the form of an ontological system, which divides levels (domains) and distinguishes highcohesion components in the form of separate ontologies of various types.

$\mathrm{S}=\langle$ Ometa, $\{$ Odomain $\}$, If $\rangle$

Ometa - meta-ontology $\langle\mathrm{X}, \mathrm{R}, \varnothing\rangle$

\{Odomain - a set of domain ontology $\langle\mathrm{X}, \mathrm{R}, \mathrm{F}\rangle$ and the ontology of software tasks $\langle\mathrm{X}, \varnothing, \varnothing\rangle$

If - is the inference engine, associated with $\mathrm{S}$.

The operation of an inference engine is characterized by the description of background situation, the determination of goal situation, and the output to the network by propagation of activation waves from background situation nodes that use the properties of relationships, associated with them.

The formal model of the resulting ontology of IS of the LPM domain knowledge can be presented in the form of a tuple of sets.

$\mathrm{O}=\langle\mathrm{X}, \mathrm{R}, \mathrm{F}\rangle$

$\mathrm{X}=\langle\mathrm{N}, \mathrm{A}, \mathrm{E}\rangle$

$\mathrm{R}=\langle\mathrm{N},\{\mathrm{X}\} \mathrm{i}, \mathrm{i}=1 . . *\rangle$

$\mathrm{E}=\langle\mathrm{N}, \mathrm{P}, \mathrm{X}\rangle$

$\mathrm{A}=\langle\mathrm{N}, \mathrm{D}, \mathrm{C}, \mathrm{X}\rangle$

$\mathrm{P}=\langle\mathrm{N}, \mathrm{A}, \mathrm{V}\rangle$

$\mathrm{D}=\langle\mathrm{N}$, meta-description, $\mathrm{F}\rangle$

$\mathrm{V}=\langle\mathrm{D}, \mathrm{C}$, meaning, $\mathrm{F}\rangle$

$\mathrm{N}=\langle$ name in language $\mathrm{L}\rangle$

$\mathrm{L}=\langle$ language $>$

$\mathrm{F}=\langle$ interpretation functions $\rangle$

$\mathrm{C}=\langle\mathrm{F}\rangle$

The most important element of the ontology model is the concept. Each concept of the $X=\{x i \mid i=1 . . N X\}$ set is assigned a three-tuple of names, attributes, and instances of concepts. The presence of $\mathrm{E}$ instances in this tuple allows defining the concept as a separate class via a set of its constituent instances.

The creation of an ontology begins with the description of the class hierarchy of notions that constitute any given domain knowledge.

The development of an ontology includes: 
- the determination of the ontology domain knowledge; their connections

the determination of domain knowledge objects and

- the organization of classes (objects) into a hierarchy:

- $\quad$ basic class $\rightarrow$ subclass;

- the formation of frames to describe classes and subclasses via definition of slots (class properties);

- filling of slots with values (creation of class

instances);

- graphical representation of the ontology.
The Protégé ontology editor allows developing ontologies as semantic webs and creating knowledge bases by the frame model, as well as forming various user requests to these bases with a view to satisfying their information needs.

After describing all classes, properties, limitations, and objects of the domain knowledge, one obtains a complex hierarchy system, which is the basis for the construction of program systems, capable of performing operations of a certain intellectual level on information, contained in the ontology. The ontology of the learning process management (Learning_process_managment) consists of the "Structure" and "Users" classes.

The graphical representation of the "LPM HEI" project ontology is presented in Fig. 1.

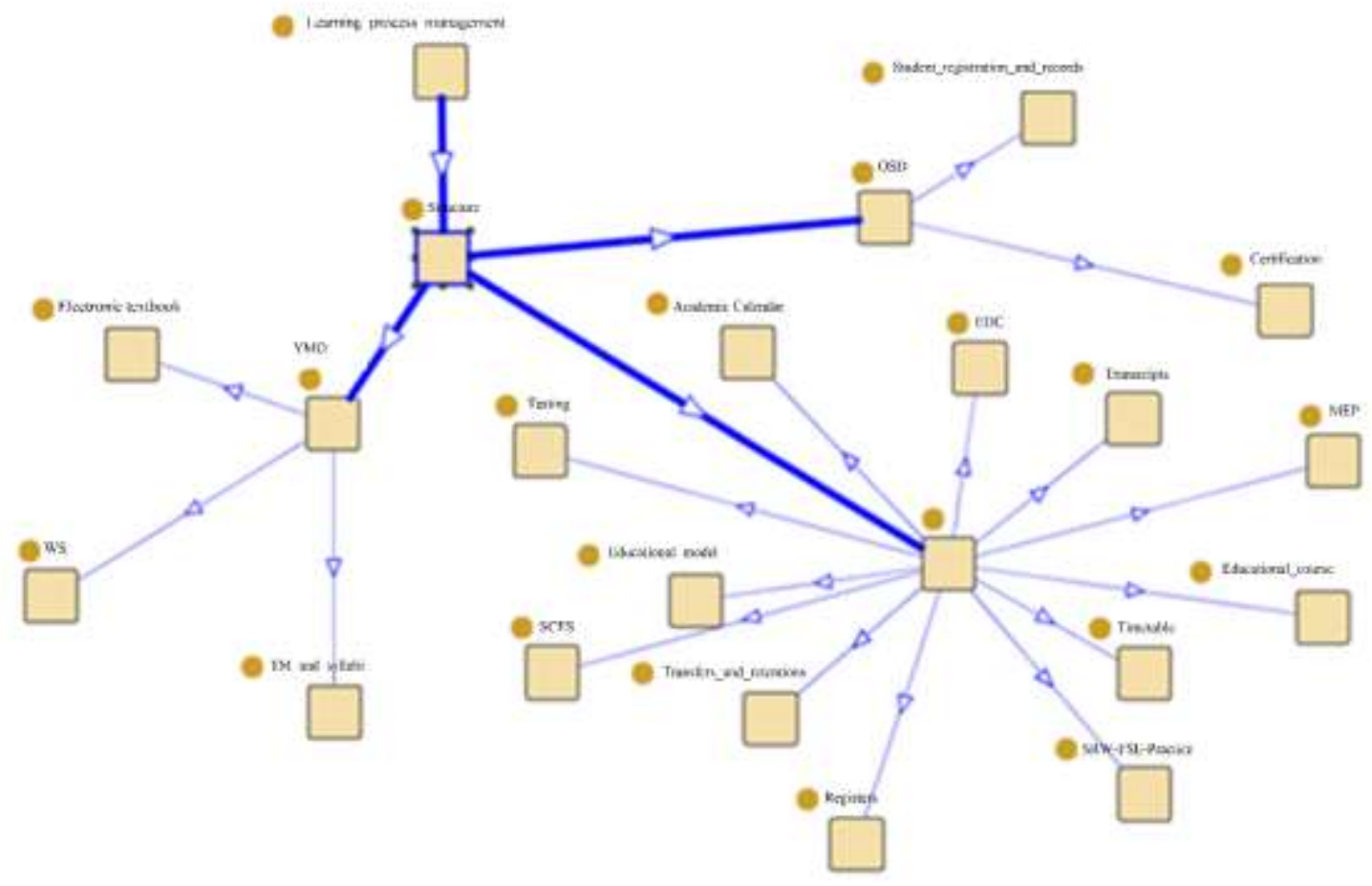

Figure 1:. Graphical representation of the "LPM HEI" project ontology.

The "Structure" class contains the structure of learning process management and the services, provided by them. The "Structure" class consists of three subclasses: "OR", "YMO", and "OSD".

The "OR" subclass is intended for the organization of control and evaluation of students' academic performance, management of processes, related to the registration of students' academic performance, estimation of their academic rating, and improvement of the credit-based technology of education. The architecture of the "OR" subclass consists of: Educational model, SCES, EDC, MEP, Academic Calendar, Educational course, Contingent, Testing, Record-books, Transcripts, Transfers and retentions, SRW-FSEPractice, Timetable, Registers.

The "OSD" subclass is intended for the organization of registration, checking, maintenance, and storage of students' personal files, preparation of documents on education. The architecture of the "OSD" subclass consists of: WS, TM and syllabi, Electronic textbook.

The "YMO" subclass is intended for the organization of learning and methodological provisions, recommendations, regulatory and reference documents on the organization and improvement of the educational process. The architecture of the "YMO" subclass consists of: Certification, Student registration and records.
The "Users" class consists of users who use the services of learning process management. The "Users" class consists of four subclasses: "User ovis registrator", "User tutor", "User student", "User parent", and "User adviser".

The "User ovis registrator" subclass is the user that registers the entire history of students' academic performance. The office registrar plans and controls the organization of the learning process.

The "User tutor" subclass is the teacher, who acts as an academic consultant in learning a specific discipline.

The "User student" subclass is a person, properly enrolled in an educational institution to receive higher or postgraduate education in accordance with a specific educational program. These include students, master's degree students, and doctoral candidates.

The "User parent" is a user, who views the academic performance of a student, i.e. the parents or guardian of a child.

The "User adviser" subclass is the teacher, acting as an academic adviser in a respective specialty, who helps choose a learning course (form an individual learning plan) and master the educational program during learning.

The knowledge base is the central part of the expert system. It contains rules that describe relationships and phenomena, methods and knowledge for the completion of objectives in the field of 
system application. One can image a knowledge base as consisting of actual knowledge and knowledge, used for the inference of other knowledge. The designed ontology will constitute the fundament of the knowledge base, which is part of the expert system for forming an automated information system. Therefore, it is necessary to search for areas of use of the knowledge base in specific domain knowledge. The following table presents question, which the ontology-based knowledge base has to answer.

Table 1:. Questions for the "User ovis_registrator" class slots.

\begin{tabular}{|l|l|}
\hline Slots & Questions \\
\hline Educational model & $\begin{array}{l}\text { How satisfied are you with the capabilities of the system for editing data on the university and the construction of the } \\
\text { educational model? }\end{array}$ \\
\hline SCES & Evaluate the possibility of viewing and editing the SCES (state compulsory educational standard). \\
\hline EDC & Evaluate the possibility of viewing and editing the EDCs (elective discipline catalog). \\
\hline MEP & Evaluate the possibility of viewing and editing the MEPs (modular educational programs). \\
\hline Academic Calendar & Evaluate the possibility of viewing and editing the academic calendars. \\
\hline WS & Evaluate the possibility of viewing and editing the WS (working syllabi). \\
\hline TM and syllabi & How satisfied are you with the capabilities of the system for viewing TM (teaching materials) and syllabi of disciplines? \\
\hline Educational course & Evaluate the possibility of viewing and editing the educational course. \\
\hline Electronic textbook & $\begin{array}{l}\text { How satisfied are you with the capabilities of the system for viewing the list of students, who subscribed to electronic } \\
\text { textbooks? }\end{array}$ \\
\hline Testing & Evaluate the capabilities of working with the testing system. \\
\hline Transcripts & Evaluate the work of the system for forming transcripts for each student. \\
\hline Transfers and retentions & How satisfied are you with the capabilities of the system for preparing documents on retentions and transfers of students. \\
\hline SRW-FSE-Practice & $\begin{array}{l}\text { How satisfied are you with the capabilities of the system, related to the SRW (scientific research work) and FSE (final } \\
\text { state examination) practice? }\end{array}$ \\
\hline Timetable & How satisfied are you with the capabilities of the system for forming the timetable of students and tutors? \\
\hline Record books & How satisfied are you with the capabilities of the system for managing the data in students' record books? \\
\hline Certification & How satisfied are you with the capabilities of the system for preparing documents on certification? \\
\hline $\begin{array}{l}\text { Student registration and } \\
\text { records }\end{array}$ & $\begin{array}{l}\text { How satisfied are you with the capabilities of the system for the organization of registration, checking, maintenance, and } \\
\text { storage of students' personal files? }\end{array}$ \\
\hline Registers & Evaluate the possibility of viewing and editing students' registers with grades. \\
\hline
\end{tabular}

The inference engine is a systematic means of proving that a certain conclusion follows from a set of assumptions. This systematic method is encoded in the inference rules, which specify the accepted logic of obtaining a conclusion. The inference is carried out by searching and comparing to the standard. Other languages require writing specific rules of search and comparison to the standard. In Turbo Prolog, these tasks are accomplished by internal unification programs; therefore, in this case, one should only write necessary specifications. Both in rule-based and logic-based systems, user receives answers to their requests in accordance with the logic, established in the system. In the first case, user requests are transformed into a form, comparable to the form of knowledge base rules. The inference engine initiates the comparison, starting with the "upper" rule. The reference to the rule is called a "call". A call of respective rules during comparison continues until the match is made or the entire knowledge base is exhausted without any matches. In the second case, transformed requests are values that are compared to values in the knowledge base.

If the inference engine finds out that it can call more than one rule, then a certain choice has to be made. At that, priority is given to the rules that are more specified or rules that include a greater volume of continuous data. This process is called conflict resolution.

The user interface system provides interaction between the expert system and the user. This interaction generally includes several functions:

1. Processing of data, obtained from the keyboard, and display of input and output data on the screen.

2. Support of a dialog between the user and the system.

3. Recognition of a misunderstanding between the user and the system.

4. Assurance of user-friendliness.

The user interface system should be capable of efficiently processing input and output. To this end, it is necessary to process input and output data quickly, clearly, and expressively. It is also necessary to include the possibility of work with additional means, such as printing devices, magnetic disks, and additional data files.

In order to perform an expert analysis, scientists developed an automated information system (AIS) with the XML universal markup language, based on the constructed ontology of the domain knowledge. Six operating modes were provided for entering the program: administrator, office registrar, teacher, advisor, student, and parent. Five of the suggested modes of entry, except for the "administrator" mode, include a test consultation of this or that expert in a respective area. All answers to test questions, obtained in this or that mode, are stored in a database, available only to the user with the right to enter the system in the "administrator" mode.

In modern conditions, the system of higher education faces many problems, the solution to which requires the use of information technologies. Implementing information technologies allows achieving efficient management and functioning of higher educational institutions. Practice shows that automatic gathering of data, their processing and storage are necessary not only for the financial and economic management of the activity of a higher educational institution. Information technologies frequently serve as the basis for the management and support of the learning process.

The number of higher educational institutions that have their own websites is constantly growing. This allows posting information, related to the higher educational institutions, and keeping in touch with students, teachers, scientists, entrepreneurs, and all those who are interested in the activity of the higher educational institution. Furthermore, remote education has become popular. This form of education allows obtaining knowledge in any place in the world at any time. This laid the foundation for the concepts of "virtual university" and "digital university" $[1,7,10]$.

Thus, information technologies are a powerful instrument for solving tasks related to managing a higher educational institution, while their implementation is irreversible. Expert methods are used to make decisions in the management systems of higher educational institutions. Expert evaluation methods are methods of organizing 
the work with expert specialists and the processing of experts' opinions [3].

There are many methods of processing expert evaluations. Mathematical and statistical methods of processing expert evaluations are intended to improve the validity of results of expert evaluation of quality. They are divided into four method subgroups: ranking, direct evaluation, consecutive advantages, and paired comparison [3].

Thus, almost all methods, used to accomplish managerial tasks in higher educational institutions, require automation. The functions of learning management systems (LMS) are implemented in the following information systems (IS): the OROKS system of the National Research University of Electronic Technology [5], the IS of the Moscow State Industrial University [5], the "Dekanat" (Deanery) information and analytical center (IAS), the IAS for control and evaluation of students' academic performance, the IAS for management of the academic load of the Cherkasy State Technological University [11], the IS of the Novgorod State University [5].

\section{Results}

In order to develop adequate factors that affect the learning process management in higher educational institutions, it is necessary to evaluate the intensity and importance of different indicators' influence. The need for distinguishing dominating indicators is determined by such peculiarities of the learning process in higher educational institutions as the multifaceted nature of the learning activity, and a large number of factors that affect the learning process and determine the quality of the result.

The analytic hierarchy process (AHP) is suggested to complete the objectives, set in this paper. The analytic hierarchy process provides for a synthesis of multiple judgments, obtainment of criteria priority and finding alternative solutions [9].

Expert procedures, based on the analytic hierarchy process, allow considering hidden connections between indicators by using experience, knowledge, and intuition of specialists in the domain knowledge, and forming a list of indicators that reflect the most important aspects of the learning process.

The authors suggest forming and determining the dominating classes of indicators that characterize the process of managing the learning process of a higher educational institution by the following stages:

1. Formation of an expert evaluation questionnaire and processing of questionnaire results.

Involving experts is necessary to gather data in accordance with the questionnaire. An expert is a qualified specialist, who meets the requirements of professional and qualimetric competence, interest in the participation in the work, businesslike character, and objectivity. An expert group is a team of specialists, organized for an expert evaluation. Members of the working group have to answer the question: how many experts are needed - one or several, do they have to be insiders or involved from the outside, who will check their level of qualification?

Headed by the unit head, the working group has to analyze and weigh all circumstances of the learning process management in a higher educational institution, and decide whether it is justifiable to rely on the opinion of a single person, and whether or not there are substantiated reasons for trusting their opinion. It is very important to avoid a large share of subjectivism during the obtainment of the evaluation. If such an expert works at the higher educational institution, he can be assigned an expert upon the approval by the administration. Invitation of an outside expert will raise the same questions of trust, and will create additional difficulties of choosing an expert and funding his activity.

An expert group can be formed in order to obtain more objective evaluation. The formation of the group consists of determination of its structure, occupational structure, number of experts, and the procedure of their selection. The occupational structure of specialists who are members of the expert group should ensure a comprehensive analysis of the task due to be solved. The group experts should have a clear understanding of the purpose and objectives of the evaluation of technological update, meet requirements to competence, interest in work, businesslike character, and objectivity. An expert's competence means the competence in the issue due to be evaluated (professional competence) and the evaluation methodology (qualimetric competence). Interest depends on the expert's individual peculiarities, stress of main work, and the possibility of using its results in their practical activity. The expert's businesslike character means their composure, working efficiency and sound decisions. The expert's objectivity means their ability to make unbiased judgments. Another task of the working group is the polling of experts. The poll can be in groups or individually, and conducted simultaneously or in several rounds. The multi-round procedure increases the objectivity of the evaluation, but requires more time and funds. At a group poll, both the group and its parts are polled simultaneously. At an individual poll, each expert is polled separately.

Based on the obtained expert evaluations, the matrices of paired comparisons are formed, which are marked as follows:

$$
C_{l}=\left(c_{i j}^{l}\right)
$$

where $l$ is the indicator number, which is part of indicator group $l$ . The number of obtained matrices matches the number of set elements of all $\tilde{K}$ indicators.

The $\mathrm{C}_{1}$ matrix aggregates experts' opinions on the issues of priority of indicators of group $\left(U_{i} i=\overline{1, l}\right)$. Matrix elements are formed as follows:

$$
C_{l}= \begin{cases}1 & \text { identical class importance } \\ 3 & \text { prevalence of class m over class n } \\ 5 & \text { significant prevalence } \\ 7 & \text { strong prevalence } \\ 9 & \text { greatest prevalence } \\ 2,4,6,8 & \text { intermediate values } \\ & \text { inverse values }\end{cases}
$$


2. Ranking of evaluation indicators and their respective groups by priority.

The ranking of indicators within a class is carried out based on a local priority vector, obtained by processing the $C_{l}$ matrices of paired comparisons.

Based on the eigenvector method, it is necessary to determine the eigenvector evaluation for each obtained matrix of paired comparisons, which would correspond with the maximum eigenvalue of the $C_{l}$ matrix.

The evaluation of eigenvector components, according to Saaty [9], is determined by approximation by formula:

$\sigma_{i}^{l}=\sqrt[m]{\prod_{j=1}^{m} c_{i j}^{l}}, \forall i=(\overline{1, m})$,

where $m$ is the dimension of the matrix (number of indicators in the group), $i$ is the row number of matrix $C_{l}, c_{i j}^{l}$ is the paired evaluation of advantages of indicators in group $l$.

The evaluation of the local priority vector is determined as follows:

$$
\alpha_{i}^{l}=\frac{\sigma_{i}^{l}}{\sum_{i=1}^{m} \sigma_{i}^{l}}, \forall i=(\overline{1, m}),
$$

where $\alpha_{i}^{l}$ is the importance (local priority) of indicator $i$ in group $l$.

Thus, the indicators of each class are compared to one another, the most significant indicators within classes are distinguished, and the

dominating indicators, with account for the class weight, are determined.

Based on the obtained information, a set of main indicators, which affect the quality of the learning process in the higher educational institution, is formed based on the adequate implementation of all its constituents.

\section{Evaluation of expert opinion consistency.}

The next stage involves the evaluation of consistency of expert evaluations [9]. In order to evaluate the consistency, it is necessary to estimate the maximum eigenvalue of the $C_{l}$ matrix.

$\lambda_{\max }^{l}=\sum_{i=1}^{m}\left(\sum_{j=1}^{m} c_{i j}^{l}\right) \cdot \alpha_{i}^{l}$,

where $\lambda_{\max }^{l}$ is the estimation of the maximum eigenvalue of the $C_{l}$ matrix.

Next, it is necessary to estimate the $O S^{l}$ consistency in accordance with (Saaty, 1993). The permissible value of the consistency relation is approximately $10 \%$.

$O S^{l}=\frac{I S^{l}}{S S_{m}}, I S^{l}=\frac{\lambda_{\max }^{l}-m}{m-1}$,

where $I S^{l}$ is the consistency index; $S S_{m}$ is the statistical consistency, which depends on the dimension of the matrix (Table 2).

Table 2:. Statistical consistency of expert opinions

\begin{tabular}{|l|l|l|l|l|l|l|l|}
\hline $\begin{array}{l}\text { Matrix dimension } \\
\mathrm{m}\end{array}$ & 1 & 2 & 3 & 4 & 5 & 7 & 6 \\
\hline$S S_{m}$ & 0.00 & 0.00 & 0.58 & 0.90 & 1.12 & 1.24 \\
\hline $\begin{array}{l}\text { Matrix dimension } \\
\mathrm{m}\end{array}$ & 9 & 10 & 11 & 12 & 13 & 1.32 \\
\hline$S S_{m}$ & 1.45 & 1.49 & 1.51 & 1.48 & 1.56 & 15 & 1.57 \\
\hline
\end{tabular}

If the matrix turns out to be inconsistent, i.e. $O S^{l}>10 \%$, then the experts have to reevaluate the indicators. In practice, however, this happens rarely, since the number of indicators is small (less than five) and the experts are generally unanimous in their decisions.

This results in the formation of weight coefficients $\alpha_{i}$ for each group of learning process evaluation indicators.

While using the analytic hierarchy process, which implies the use of paired comparison matrices, it is necessary to evaluate not only the consistency of each expert's matrices, but also the opinion consistency of the group of experts. Approaches, based on the move from paired comparisons to ranking and subsequent estimation of Kendall's rank correlation coefficients, concordance or estimation of the priority vector do not provide for an evaluation of consistency of matrices themselves, i.e. primary information is lost. This paper suggests using the paired comparison inconsistency coefficient [8]:

$k_{i j}=\widehat{q_{l \jmath}}\left|\log _{r} \frac{a_{1 i j}}{a_{2 i j}}\right|$, where $k_{i j}$ is the inconsistency coefficient of paired comparisons; $\widehat{q_{l]}} \in[0,0.5]$ is the weight coefficient that determines the inconsistency between expert evaluations; $a_{1 i j}$ is the result of comparison of element $\mathrm{i}$ of hierarchy $\mathrm{j}$ by the $1^{\text {st }}$ expert, $a_{2 i j}$ is the result of comparison of element $i$ of hierarchy $j$ by the $2^{\text {nd }}$ expert; $r$ is the relative importance scale for the analytic hierarchy process, which equals nine.

The coefficient allows evaluating the consistency of paired comparison matrices at the level of elements, accounts for transitivity and cardinality of advantages of specialists' judgments, and includes the information on the competence of experts.

The evaluation of a highly competent expert traditionally is more important and influential to the generalized evaluation. This allows eliminating inconsistencies between experts in favor of the most competent one among them, levelling the evaluation of his colleague who is not as competent. $\widehat{q_{l j}}$ reaches maximum if $q_{1 i j}=q_{2 i j}$, i.e. both experts are equally competent and it is impossible to ignore the opinion of either of them. Thus, the smaller is the difference between 
the experts' competence levels, the more important is the inconsistency between their opinions.

The condition for the consistency of expert opinions and the integration of two paired comparison matrices is:

$\bar{k}_{J}=\frac{1}{n-1} \sum_{i=1, i \neq j}^{n} k_{i j} \leq 0,315, j \in[1 ; n]$,

If with a certain value of $j$ this condition is not met, then this shows significant inconsistency between experts in the evaluation of element $j$ of the hierarchy; therefore, such matrices cannot be integrated.

In order to obtain the general opinion of experts regarding a certain question, it is necessary to check conditions (7) for each pair of experts. This check will result in the grouping of all experts by the consistency of their judgments. Four main variants are possible: all experts fall within one group; 2-3 groups of like-minded persons are formed; many small groups are formed; almost all experts fail to fall within any group. In the first case, the judgments of experts who did not fall within the general group are ignored. In the second case, it is necessary to examine the generalized opinion of each formed group and then reintegrate the formed matrices. In the third and fourth cases, it is necessary to check thoroughly the conditions of the examination for mistakes. Then it is necessary to conduct a reexamination, if possible, or return to the stage, during which the mistake was made.

The generalized opinion of the group experts is expressed in the form of a generalized matrix of paired comparisons:

$\overline{a_{l j}}=\sqrt[\sum_{l=1}^{k} q_{l i j}]{\prod_{l=1}^{k} a_{l i j}^{q_{l i j}}}$,

where $\left\{a_{i j}\right\}$ is the matrix of paired comparisons of $n$ elements of the hierarchy by the $1^{\text {st }}$ expert; $\left\{q_{i j}\right\}, l \in[1 ; \mathrm{k}], \mathrm{i} \in[1 ; \mathrm{n}], \mathrm{j} \in[1 ; \mathrm{n}]$ is the competence matrix of expert 1 , the $q_{i j}$ element of which is the competence coefficient of the expert during the comparison of element $i$ of the hierarchy with element $j$, at that, $q_{i j}=q_{j i}$ and $q_{i j} \in[0$; $1]$.

4. Graphical representation of obtained priorities, analysis and interpretation of results.

The set of indicators of the learning process evaluation has the following weight coefficients (Fig. 2.).

The "Inconsistency" indicator is low ( $B O \leq 0,1$ ), which confirms the consistency of expert evaluations.

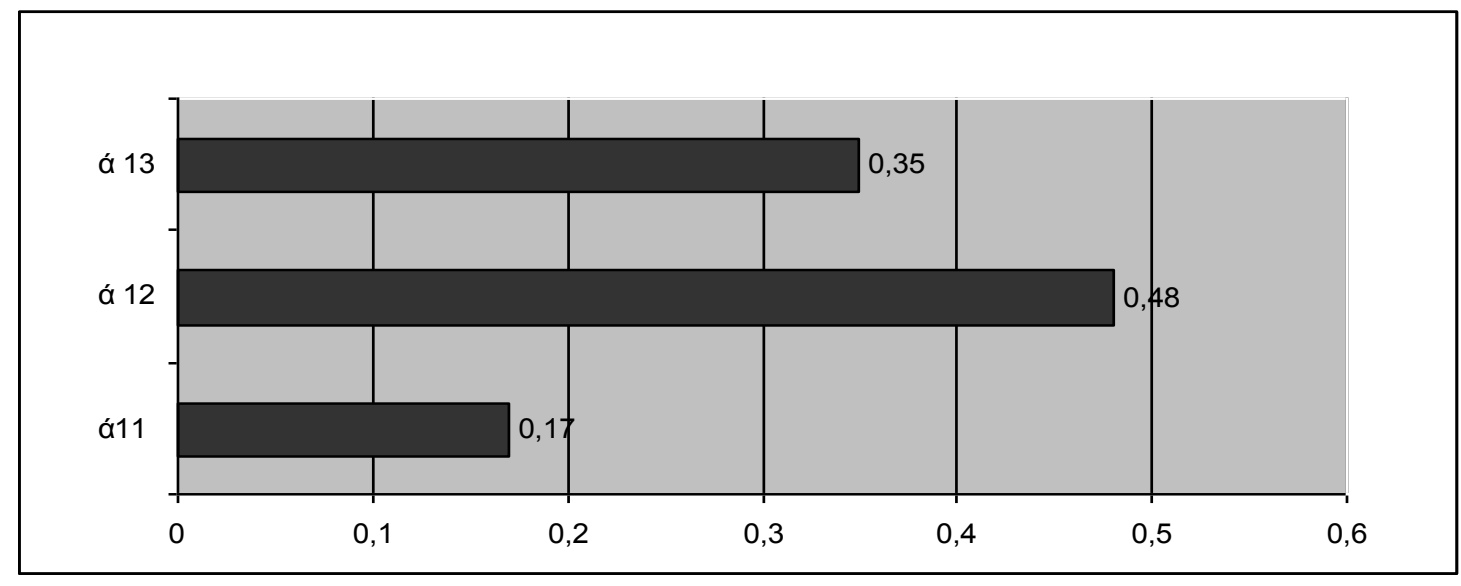

Figure 2:. Ranking of the group of learning process evaluation indicators

The second group of indicators received the following weight coefficients (Fig. 3.)

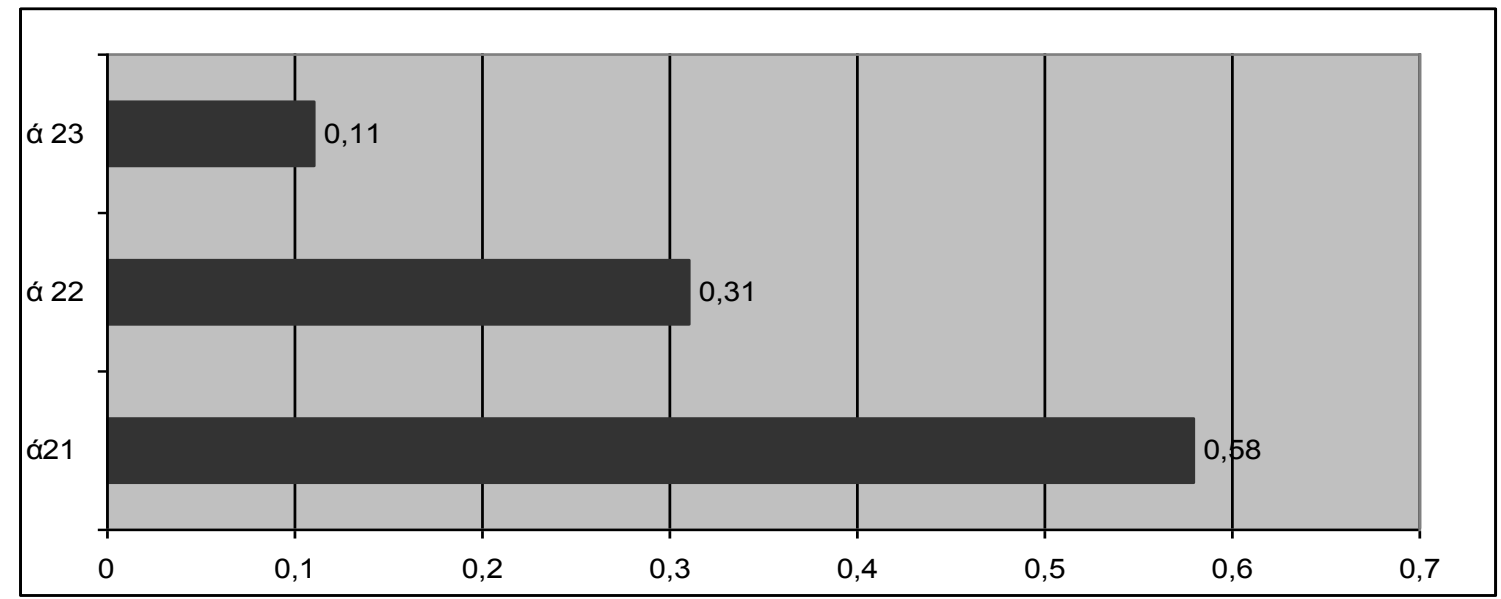

Figure 3:. Weight coefficients of the second group of indicators. 
The estimation of the inconsistency coefficient for two paired comparison matrices is presented in the Table 3. For each pair of indicators $k_{I E R, I I R}=0,09227<0,315 ; \quad k_{I E R, L S C R}=0,116241<0,315$; $k_{I I R, L S C R}=0<0,315$, thus, no inconsistency between the first and second expert was found. The analysis showed that all paired comparison matrices for each expert are consistent. This is an additional confirmation of the fact that experts are highly qualified specialists.

Table 3:. Expert evaluation consistency

\begin{tabular}{|c|c|c|c|}
\hline \multicolumn{4}{|c|}{ Paired comparison matrix of the $1^{\text {st }}$ expert } \\
\hline & IER & IIR & LSCR \\
\hline IER & 1 & 0.5 & 0.33 \\
\hline IIR & 2 & 1 & 2 \\
\hline LSCR & 3 & 0.5 & 1 \\
\hline \multicolumn{4}{|c|}{ Paired comparison matrix of the $2^{\text {nd }}$ expert } \\
\hline & IER & IIR & LSCR \\
\hline IER & 1 & 0.33 & 0.2 \\
\hline IIR & 3 & 1 & 2 \\
\hline LSCR & 5 & 0.5 & 1 \\
\hline$k_{I E R, I I R}=0.09227$ & \multicolumn{2}{|c|}{$k_{I E R, L S C R}=0.116241$} & \\
\hline
\end{tabular}

Thus, the weight coefficients of the comprehensive evaluation system can be considered adequate, since the conditions of paired comparison matrices' integration is met. Almost all experts were unanimous, while eight of them could be united into one group.

The conduction of the expert evaluation resulted in the use of the following methods:

I. Creation of groups. A working group was created for the organization of the expert evaluation. The functions of this group included polling, processing of materials, and analysis of results of the collective expert evaluation. The working group assigned experts from higher educational institutions, who helped get answers to the set questions regarding the activity of the automated information system of learning process management of this or that higher educational institution. The number of experts, involved in the development of the prediction, was 150 persons from three higher educational institutions.

II. Development of a questionnaire. This method involves the development of questions regarding the automated information system of learning process management of this or that higher educational institution, which were suggested to the experts. The questionnaire form was developed with the XML universal markup language. At that, the questions were compiled according to a certain structural and hierarchic scheme, i.e. from the broader questions to the narrower ones, from complicated questions to the simple ones.

III. Estimation of expert evaluations. The processing of materials of expert evaluations, characterizing the generalized opinion and level of consistency and experts' individual evaluations. The processing of expert evaluation data is the source material for the activity of an automated information system of learning process management of this or that higher educational institution.

A score, ranging from 0 to 1.0 was assigned in order to determine the importance of a parameter. In this case, the importance of a parameter is determined by formula:

$\mathrm{a}_{\mathrm{i}}=\left[\left(\mathrm{P}_{\mathrm{ij}}: \mathrm{P}_{\mathrm{cj}}\right)\right] / \mathrm{m}$,

were $a_{i}$ is the importance of object parameter $i ; i$ is the number of the object parameter; $j$ is the expert number; $m$ is the number of experts in the group; $P_{i j}$ is the score, assigned to parameter $i$ by expert $j ; P_{c i}$ is the sum of scores, assigned by expert $j$ to all object parameters.

The importance of other object parameters is determined in a similar fashion. The importance of parameters is determined as follows. First, a paired correlation between parameters is found for each expert. If the importance of a given parameter, according to the expert, is greater than that of another one, with which this parameter is compared, then this parameter is assigned two points. If the importance of the parameters is identical, the parameter is assigned one point. If the importance of the parameter of smaller than that of the other one, the first parameter is not assigned any points.

The final qualitative evaluation was determined by four main expert evaluation methods and a set of their varieties:

1) simple ranking method (or preference method);

2) weight coefficient method;

3) paired comparison method;

4) consecutive comparison method.

\section{Discussion}

The scientific problem, set in the paper, determined the need of using the XML universal markup language for the design.

The improvement of specialist training quality, as a key problem of the modern educational process, requires further work for the improvement of methodological approaches to the category of quality as an integral characteristic, determined by a number of properties and functional capabilities of the object, participant of the educational process.

The conducted study substantiated the fact that the need for distinguishing dominating indicators is determined by the multifaceted nature of the studied processes in the educational activity of a higher educational institution, as well as a great number of destabilizing factors that affect the quality of the learning process result. The preliminary list of factors was formed based on a retrospective analysis of the educational activity and was analyzed by experts, with a view to determining the dominating factors.

The suggested model of choosing dominating factors is based on the analytic hierarchy process. It allows distinguishing them by the level of priority for the purposes of managing a higher educational institution's learning process. The main stages of this approach are determined by the following sequence of steps:

1) forming a questionnaire for the expert evaluation and the processing of questionnaire results;

2) ranking the indicators by priority;

3) evaluating expert opinion consistency;

4) graphical representation of obtained priorities, analysis and interpretation of results.

Optimization of teaching and learning with the help of AHP was considered by Tsinidou, Gerogiannis \& Fitsilis (2010); in their works they developed the subject priority estimation criteria through paired comparison on a scale from 1 to 9 , and then matrix is to compare the results with each criteria group (faculty, administration, library, with the curriculum structure, the institute's location). But the lack of expert analysis can create an inappropriate priority scale. 
This is due to the fact that each of the respondents would prioritize based on personal motives, while priority expert will be guided by practicality and impartiality.

Badri \& Abdulla [19] also believe that determining the priorities in the educational process can improve training performance. All elements at each level should be compared in pairs, so it will be easier to determine the measure of importance for each element this idea can be traced in all three works. Still, Badri \& Abdulla [19] believe that AHP can cause some problems due to the difficulty of calculations, therefore they recommended to divide the AHP into separate stages. To date, by using modern information programs, the difficulties in training optimization through AHP can be eliminated.

\section{Conclusions}

Thus, the automation of processes in the learning activity of a modern higher educational institution should be based on the use of knowledge-based approaches. The application of ontologies allows systematizing, structuring, and reusing accumulated knowledge and experience. The developed ontology describes the thesaurus of the domain knowledge, in particular, determines the concepts of the higher educational institution's structure and describes various types of users. The ontology is the basis for the development of a knowledge base for the learning process management. The Protégé ontology editor, on the one hand, allowed using international standards, while on the other hand, provided a convenient user interface during the construction of the ontology. The obtained ontology can be united with other ones, for example, those, described in other information systems of a higher educational institution, and expanded by accumulating facts.

The developed technology allows using expert experience and knowledge, conducting expert polls, processing obtained evaluations, saving and accumulating domain knowledge. This allows using expert knowledge, stored in the knowledge base, for future decision-making, generally assuring a stable improvement of the efficiency of learning process management in higher educational institutions.

\section{References}

[1] Albaqami, N. (2014). Critical Investigation of Virtual Universities: Applying the UK Structure to Saudi Arabia. United Kingdom. Retrieved from https://www.dora.dmu.ac.uk/bitstream/handle/2086/11125/Critical Investigation of Virtual Universities.pdf?sequence $=1$ \&isAllowed $=\mathrm{y}$

[2] Bogino, V. I., Levchuk, A. N., \& Petrov, Y. G. (2009). Information technology in collaboration support during personnel training and retraining. Management Systems and Machines, 2, 66-72.

[3] Hardman, D. (2010). Thinking psychological perspectives on reasoning, judgment and decision making. Wiley.

[4] Izvozchikov, V. A. (1991). Ionosphere educology. New learning information technologies. Saint-Petersburg.

[5] Kryukov, V. V. (2007). The corporate information environment of a higher educational institution: Methodology, models, solutions. Vladivostok: Dalnauka.

[6] Maystrenko, A. V., \& Maystrenko, N. V. (2012). Information technologies in science, education, and engineering practice: study guide. Tambov: Tambov State Technical University Publishing House.

[7] Moravec, J. W. (2007). A New Paradigm of Knowledge Production in Minnesota Higher Education: A Delphi Study. ProQuest Information and Learning Company.

[8] Paderno, P. I., Burkov, Y. A., \& Yevgrafov, V. G. (2011). Paired comparison consistency criterion. Information and Management Systems, 3, 56-60.
[9] Saaty, T. L. (1993). Decision making with the analytic hierarchy process. Moscow: Radio i svyaz (Radio and communication).

[10] Shahtalebi, S., Shatalebi, B., \& Shatalebi, F. (2011). A Strategic Model of Virtual University. Procedia - Social and Behavioral Sciences, 28, 909-913. Retrieved February 23, 2016, from http://www.sciencedirect.com/science/article/pii/S1877042811026061

[11] Tymchenko, A. A., Trius, Y. V., Oksamytna, L. P., \& Stetsenko, I. V. (2009). New approaches to the creation of a system of control and evaluation of the academic performance of higher educational institutions' students. Information Technologies in Education, 4, 111123. Retrieved from http://ite.kspu.edu/ru/issue-4/p-111-123

[12] Webster F. Theories of the information society. - Routledge, 2014

[13] Liebowitz J., Frank M. (ed.). Knowledge management and e-learning. - CRC press, 2016.

[14] Moore M. G. (ed.). Handbook of distance education. - Routledge, 2013.

[15] Jones G., Sallis E. Knowledge management in education: Enhancing learning \& education. - Routledge, 2013.

[16] Rennie F., Morrison T. E-learning and social networking handbook: Resources for higher education. - Routledge, 2013.

[17] Al-Qahtani A. A. Y., Higgins S. E. Effects of traditional, blended and e-learning on students' achievement in higher education //Journal of Computer Assisted Learning. - 2013. - T. 29. - №. 3. - C. 220-234.

[18] Tsinidou, M., Gerogiannis, V., \& Fitsilis, P. (2010). Evaluation of the factors that determine quality in higher education: an empirical study. Quality Assurance in Education, 18(3), 227-244

[19] Badri, M. A., \& Abdulla, M. H. (2004). Awards of excellence in institutions of higher education: an AHP approach. International Journal of Educational Management,

[20] Button D., Harrington A., Belan I. E-learning \& information communication technology (ICT) in nursing education: A review of the literature //Nurse Education Today. - 2014. - T. 34. - №. 10. - C. 1311-1323. 